\title{
Revisiting the relevance of the 3-month safety period in the evaluation of prosthetic valve endocarditis with FDG-PET/CT
}

\author{
Daniel Juneau, MD, ${ }^{\mathrm{a}, \mathrm{b}}$ and Matthieu Pelletier-Galarneau, MD, MSc, ${ }^{\mathrm{c} d}$ \\ a Department of Medical Imaging, Centre Hospitalier de l'Université de Montréal, Montréal, QC, \\ Canada \\ b University of Ottawa Heart Institute, Ottawa, Canada \\ c Department of Medical Imaging, Institut de Cardiologie de Montréal, Montréal, Canada \\ d Gordon Center for Medical Imaging, Massachusetts General Hospital, Harvard Medical School, \\ Boston, MA
}

Received Feb 4, 2020; accepted Feb 4, 2020

doi: $10.1007 /$ s12350-020-02070-y

\section{See related article, pp. 2258-2268}

Positron emission tomography/computed tomography (PET/CT) with ${ }^{18} \mathrm{~F}$-fluorodeoxyglucose (FDG) is the workhorse of nuclear oncology. One of the main limitations of FDG-PET/CT in oncology is the nonspecific tracer uptake associated with infection and inflammation. In nuclear cardiology, this limitation has been put to good use and the role of FDG-PET/CT has drastically expended over the past years. Indeed, FDGPET/CT has proven very useful in the evaluation of inflammation related to cardiac sarcoidosis ${ }^{1,2}$ and vasculitis. $^{3}$ Furthermore, FDG-PET/CT has also been shown to play an important role in the evaluation of infection such as cardiovascular implantable electronic devices (CIED) infections. ${ }^{4,5}$

Endocarditis is a dreaded complication of prosthetic valve implantation with an incidence of $1-6 \% .^{6}$ Diagnosis of prosthetic valve endocarditis (PVE) is especially challenging given the non-specific symptoms as well as relatively poor performance of the modified Duke Criteria for prosthetic valves. Indeed, compared to native valve endocarditis, PVE is associated with higher

Reprint requests: Daniel Juneau, MD, Department of Medical Imaging, Centre Hospitalier de l'Université de Montréal, 1000 rue Saint-Denis, Montréal, QC H2X 0C1, Canada; daniel.juneau@umontreal.ca

J Nucl Cardiol 2021;28:2269-71.

$1071-3581 / \$ 34.00$

Copyright (C) 2020 American Society of Nuclear Cardiology. rates of negative blood cultures as well as higher rates of false negative echocardiography. ${ }^{6}$ FDG-PET/CT has been demonstrated to play a complementary role to echocardiography in the evaluation of PVE; the addition of abnormal FDG uptake as a new major Duke criterion improves their sensitivity significantly, without affecting their specificity, ${ }^{7}$ mainly by reducing the number of "Possible IE." These data led to the integration of FDG-PET/CT as a new major criterion for the diagnosis of PVE in the 2015 European Society of Cardiology (ESC) guidelines. ${ }^{6}$ Since then, two meta-analysis have confirmed the good sensitivity and specificity of FDGPET/CT for the diagnosis of PVE. ${ }^{8,9}$ Importantly, according to these guidelines, in order to be considered a major criterion, FDG-PET/CT has to be performed $>3$ months after valve implantation. This limitation, based mostly on case reports and expert opinions, was implemented to reduce the possibility of false positive studies caused by post-operative inflammatory response. However, despite a decrease in perioperative PVE over the past several decades, a significant proportion of PVE still occurs in the months following surgery. ${ }^{10}$ Since the publication of the 2015 ESC guidelines, new evidence has emerged to challenge the validity of this 3-month safety period. For instance, Swart et al performed a multicentre study of 160 patients with suspected PVE for the evaluation of the diagnostic performance of FDG-PET/CT. ${ }^{11}$ They showed that a high diagnostic accuracy could be achieved in the early post-operative period ( $<3$ months) and that imaging in the early postimplantation period was not a predictor of decreased diagnostic accuracy. 
In this issue of the Journal, Wahadat et al present the results of a prospective, multicentre study in which they performed FDG-PET/CT in 37 patients with recently implanted prosthetic aortic valve, with no clinical suspicion of PVE. ${ }^{12}$ Patients were divided in three groups and assigned to undergo FDG-PET/CT either 5,12 , or 52 weeks after implantation. They report mild to moderate uptake around the prosthetic heart valve (PHV) in the majority of patients, with a pattern of uptake characterized as diffuse or located along the valve struts in 34/37 patients. Importantly, there were no significant differences in $\mathrm{SUV}_{\max }, \mathrm{SUV}_{\text {ratio }}$ (PHV $\mathrm{SUV}_{\max }$ divided by mean SUV of the blood pool), or pattern of FDG uptake between the three time points. These results are concordant with those recently reported by Roque et al, ${ }^{13}$ who performed serial FDG-PET/ CT scans in patients with PHV and without clinical suspicion of PVE at 1, 6, and 12 months post implantation. Interestingly, both studies report very similar $\mathrm{SUV}_{\text {max }}$ around aortic PHV at their earliest imaging timepoint (mean $\mathrm{SUV}_{\max }$ of 4,1 in both studies). Key differences between the two studies include the fact that Roque et al also included patients with mitral PHV and that they imaged the same patients at multiple time points, whereas Wahadat et al assigned each patient to a single specific time point. Both studies reached the conclusion that there are no significant changes in intensity and pattern of FDG uptake around uncomplicated PHV between approximately 1 month and 12 month following surgery. Indeed, they both demonstrated that FDG uptake was homogeneous in the vast majority of scans and that uptake distribution was not different at the various time points, supporting the idea that uptake pattern could be used to distinguish between post-operative inflammation and infection in the early period (1 to 12 month). This is in agreement with other studies demonstrating that FDG uptake distribution (homogeneous vs heterogeneous or focal) rather than uptake intensity is useful at distinguishing normal postoperative inflammation from infection. ${ }^{14,15}$

A weakness of the current study is the fact that despite asking the patients to follow a low carbohydrate diet for 24 hours and fast for 12 hours before the scan, complete suppression of physiological myocardial uptake was achieved in only $49 \%$ of patients. The authors themselves report that this hampered the interpretation of 1 scan. It is undeniable that proper preparation is a critical and contributive technique and should be used in all patients undergoing cardiac FDGPET/CT for the assessment of myocardial inflammatory or infectious diseases. ${ }^{16,17}$ Whether or not this has a significant impact on the accuracy of FDG-PET/CT for the diagnosis of PVE remains to be shown. In the recent multicentre study by Swart et al, the quality of physiological uptake suppression was not associated with decreased diagnostic accuracy. ${ }^{11}$ However, this does not mean that proper preparation is unnecessary; it can be achieved reasonably easily, and we should strive to make the interpretation of these studies as easy and reliable as possible. What this does tell us, however, is that in real-life condition, even with poor preparation and incomplete or absent suppression, which is not uncommon, it is usually possible to accurately differentiate between normal and abnormal patterns of uptake around PVH. One thing to keep in mind is that adequate suppression might also be more important in certain locations. For instance, evaluation of mitral valves might be more hindered by poor suppression compared to aortic valves given the typical distribution of physiological myocardial uptake surrounding the basal segments of the left ventricle. Further studies will be required to elucidate these questions

Abnormal FDG-PET/CT uptake around a PHV was introduced as a major criterion for the diagnosis of PVE in the ESC guidelines of 2015. Since then, retrospective and prospective studies have challenged the relevance of the arbitrary 3-month post-operative waiting period. By focusing interpretation of FDG-PET/CT studies on uptake distribution and pattern rather than uptake intensity, distinction between post-surgical inflammation and infection appears possible as soon as 1 month after surgery. Hopefully, the addition of FDG-PET/CT imaging in the workup of these patients will help us to improve their outcomes, particularly in the critical early post-operative period.

\section{Disclosures}

D.J. has received consultant fees from AbbVie and Advanced Accelerator Applications. M.P.-G. has no disclosures.

\section{References}

1. Chareonthaitawee P, Beanlands RS, Chen W, et al. Joint SNMMIASNC expert consensus document on the role of 18F-FDG PET/ $\mathrm{CT}$ in cardiac sarcoid detection and therapy monitoring. J Nucl Med 2017;58:1341-53.

2. Martineau P, Pelletier-Galarneau M, Juneau D, Leung E, Birnie D, Beanlands RSB. Molecular imaging of cardiac sarcoidosis. Curr Cardiovasc Imaging Rep 2018;11:6.

3. Pelletier-Galarneau M, Ruddy TD. PET/CT for diagnosis and management of large-vessel vasculitis. Curr Cardiol Rep 2019;21:34.

4. Sarrazin J-F, Philippon F, Tessier M, et al. Usefulness of fluorine18 positron emission tomography/computed tomography for identification of cardiovascular implantable electronic device infections. J Am Coll Cardiol 2012;59:1616-25.

5. Kim J, Feller ED, Chen W, Dilsizian V. FDG PET/CT imaging for LVAD associated infections. JACC Cardiovasc Imaging 2014;7:839-42. 
6. Habib G, Lancellotti P, Antunes MJ, et al. ESC Guidelines for the management of infective endocarditis: The Task Force for the Management of Infective Endocarditis of the European Society of Cardiology (ESC). Endorsed by: European Association for CardioThoracic Surgery (EACTS), the European Association of Nuclear Medicine (EANM). Eur Heart J 2015;36:3075-128.

7. Saby L, Laas O, Habib G, et al. Positron emission tomography/computed tomography for diagnosis of prosthetic valve endocarditis: increased valvular 18F-fluorodeoxyglucose uptake as a novel major criterion. J Am Coll Cardiol 2013;61:2374-82.

8. Mahmood M, Kendi AT, Ajmal S, et al. Meta-analysis of $18 \mathrm{~F}-$ FDG PET/CT in the diagnosis of infective endocarditis. J Nucl Cardiol 2019;26:922-35.

9. Juneau D, Golfam M, Hazra S, et al. Molecular Imaging for the diagnosis of infective endocarditis: A systematic literature review and meta-analysis. Int J Cardiol 2017;253:183-8.

10. Piper C, Korfer R, Horstkotte D. Prosthetic valve endocarditis. Heart 2001;85:590-3.

11. Swart LE, Gomes A, Scholtens AM, et al. Improving the diagnostic performance of $18 \mathrm{~F}$-fluorodeoxyglucose positron-emission tomography/computed tomography in prosthetic heart valve endocarditis. Circulation 2018;138:1412-27.

12. Wahadat AR, Wi Tanis, Scholtens AM, et al. Normal imaging findings after aortic valve implantation on 18F-fluorodeoxyglucose positron emission tomography with computed tomography. J Nucl Cardiol 2020;2:85. https://doi.org/10.1007/s12350-019-02025-y.

13. Roque A, Pizzi MN, Fernández-Hidalgo N, et al. Morpho-metabolic post-surgical patterns of non-infected prosthetic heart valves by $[18 \mathrm{~F}]$ FDG PET/CTA: "normality" is a possible diagnosis. Eur Heart J Cardiovasc Imaging 2020;21:24-33.

14. Mumme T, Reinartz P, Alfer J, Müller-Rath R, Buell U, Wirtz D. Diagnostic values of positron emission tomography versus triplephase bone scan in hip arthroplasty loosening. Arch Orthop Trauma Surg 2005;125:322-9.

15. Hariri H, Tan S, Martineau P, et al. Utility of FDG-PET/CT for the detection and characterization of sternal wound infection following sternotomy. Nucl Med Mol Imaging 2019;53:253-62.

16. Tang R, Wang JT-Y, Wang L, et al. Impact of patient preparation on the diagnostic performance of $18 \mathrm{~F}-\mathrm{FDG}$ PET in cardiac sarcoidosis: A systematic review and meta-analysis. Clin Nucl Med 2016;41:e327-39.

17. Dilsizian V, Bacharach SL, Beanlands RS, et al. ASNC imaging guidelines/SNMMI procedure standard for positron emission tomography (PET) nuclear cardiology procedures. J Nucl Cardiol 2016;23:1187-226.

Publisher's Note Springer Nature remains neutral with regard to jurisdictional claims in published maps and institutional affiliations. 